\title{
Secrecy-Capacity-Optimization-Artificial-Noise in Large-Scale MIMO: Ergodic Lower Bound on Secrecy Capacity and Optimal Power Allocation
}

\author{
Yebo Gu $\mathbb{D}^{D}$, Zhilu Wu $\mathbb{D}^{D}$, and Zhendong Yin \\ School of Electronics and Information Engineering, Harbin Institute of Technology, Harbin, China \\ Correspondence should be addressed to Zhilu Wu; 16b305002@hit.edu.cn
}

Received 2 June 2021; Revised 19 July 2021; Accepted 2 August 2021; Published 19 August 2021

Academic Editor: Hongyuan Gao

Copyright ( $\odot 2021$ Yebo Gu et al. This is an open access article distributed under the Creative Commons Attribution License, which permits unrestricted use, distribution, and reproduction in any medium, provided the original work is properly cited.

\begin{abstract}
The security of wireless information transmission in large-scale multi-input and multioutput (MIMO) is the focus of research in wireless communication. Recently, a new artificial noise-SCO-AN which shows no orthogonality to the channel, is proposed to overcome the shortcomings of traditional artificial noise. In the previous research, the optimization function of SCO-AN is not convex, and its extremum cannot be obtained. Usually, nonconvex optimization algorithms or iterative relaxation algorithms are used to get the maximum value of the optimization objective function. Nonconvex optimization algorithms or iterative relaxation algorithms are greatly affected by the initial value, and the extremum cannot be obtained by a nonconvex optimization algorithm or iterative relaxation algorithm. In this paper, we creatively apply the strong law of large numbers to obtain the optimal value of the optimization function of SCO-AN under the condition of large-scale MIMO: the strong law of large numbers is applied to obtain the ergodic lower bound (ELB) expression of SC for SCO-AN. The power allocation (PA) problem of the SCO-AN system is discussed. We use a statistical method to get the formula for calculating the optimal power distribution coefficient of the SCO-AN system. The transmitter can use the optimal power ratio of PA to distribute the transmitted power without using the PA algorithm. The effect of imperfect channel state information is discussed. Through simulation, we found that more power should be generated for SCO-AN if the channel estimation is imperfect and the proposed method can achieve better security performance in the large-scale MIMO system.
\end{abstract}

\section{Introduction}

Security is the core issue of wireless communication [1]. In this paper, the security of wireless information transmission is discussed. Since the wireless communication signal is transmitted in broadcast ways, the wireless communication signal can be received by eavesdroppers without restriction. Since the eavesdropper cannot be prevented from obtaining the transmitted information, previous research on secure communication is focused on using the principles of encryption to prevent eavesdroppers from deciphering the obtained information. However, encryption cannot fundamentally solve secure transmission problem if the eavesdropper obtains the key of the information or has enough computing power to crack the information. The secure transmission of information based on cryptography will fail. Therefore, reducing the amount of information obtained by eavesdroppers is the correct way to achieve secure communication. In recent years, physical layer security (PLS) technology has become a new research focus.

The PLS technology was proposed in [2]. In [2], a classic model for secure communication model is proposed. In [3], the secure communication model is extended to the field of wireless communication, the channels in the secure communication models are all broadcast channels.

The application of MIMO provides an opportunity for the development of PLS. In [4], the concept of secrecy capacity (SC) is outlined by Barros and Rodrigues. The numerical expression of SC is studied in [5]. In [6], Zhu studies the SC of wiretap fading channels in a massive MIMO communication system. Zeng proposes the SC of downlink massive MIMO-NOMA networks [7]. A novel method to select the 
proper antenna and PA in massive MIMO is proposed in [8], the algorithm in [8] makes the wireless communication system to adjust the PA coefficient, and the communication quality of eavesdropper is reduced effectively.

SC is determined by the capacity of legitimate and eavesdropping channels. Generally, increasing the capacity of the legitimate channel or decreasing the capacity of the eavesdropping channel could increase SC [9-11]. However, for a particular communication system, the capacity of the legitimate channel is challenging to change. Therefore, previous studies focus on reducing the capacity of eavesdropping channels [12-14].

Artificial noise (AN) is a specific application for PLS technology [15]. AN is orthogonal to the receiver's channel, so AN will not affect the legitimate channel. However, AN is not orthogonal to the eavesdropping channel; AN will affect the eavesdropping channel. Adding AN to the transmitted signals will raise the SC of secure transmission.

However, the design of $\mathrm{AN}$ is under strict restriction. $\mathbf{H}$ denotes the legitimate channel state information (CSI), and $\mathbf{w}_{k}$ denotes the AN. By definition of $\mathrm{AN}, \mathbf{H} \mathbf{w}_{k}=0 . \mathbf{w}_{k}$ is the zero solution of homogeneous linear equation group $\mathbf{H X}=$ 0 . The dimension of $\mathbf{H}$ is $m \times n$. If $\mathbf{X}$ is nonzero, the rank $(\mathbf{H})$ must be guaranteed that $\operatorname{rank}(\mathbf{H})<\min (m, n)$. It means that the number of transmitting and receiving antennas has to be different. However, transmitting antennas have the same number as receiving antennas in most MIMO real-life communication systems. We have to reduce the transmitting or receiving antennas. Meanwhile, the capacity of legitimate channel is reduce.

SCO-AN is proposed in [16] to solve the problem. SCOAN does not show orthogonality to the legitimate and eavesdropping channels. Therefore, SCO-AN is achievable in any situation. Although this method has a certain degree of negative impacts on the legitimate channel, SCO-AN has a greater degree of negative impacts on the eavesdropping channel. SCO-AN is considered to increase the SC to a greater degree when compared to the traditional AN. SCO-AN is a tool that converts the receiver's antinoise ability into SC.

Recently, intelligent reflecting surface (IRS) technology has become a research hotspot in communication technology $[17,18]$. Because the channel of IRS is divided into uplink and downlink. Artificial noise is only valid in the uplink or downlink so that AN cannot be applied to IRS. The nonorthogonal characteristic of SCO-AN makes it a perfect application in IRS. In 2020, [19] applied nonorthogonal artificial noise to improve the security performance of IRS systems. It should be pointed out that the idea of nonorthogonal artificial noise was first put forward in [16].

We should consider the transmission power limits. It is important to allocate the power of the SCO-AN system [20]. The objective function of SCO-AN PA is nonconvex. It has been proved that we cannot get the optimal PA coefficient of the SCO-AN system. In general, nonconvex optimization algorithm or relaxation iteration algorithm is used to optimize nonconvex functions [21-24]. The simulation result has proved that these two algorithms are greatly affected by initial states of the communication models. We can only adopt an iterative approach to calculate the maximum of the objective function in a certain range. However, in the massive MIMO communication system, we can use strong law of large numbers to approximate the ELB of SC. Based on the ELB of SC, the optimal PA coefficient of SCOAN is obtained as well. The expression for ELB on SC in fading channels which adds SCO-AN is derived. The optimal ratio of PA of the SCO-AN system is then deduced.

Base on the optimal PA coefficient, in a large-scale MIMO system, we can allocate the power without calculating. Furthermore, the effects of the perfect CSI and the imperfect CSI on the ELB of SC are discussed. The study of this paper is highly compatible with the features of $6 \mathrm{G}$ networks which equip massive MIMO communication system [25]. Therefore, the application introduced in this paper is a promising solution in addressing security threats for $6 \mathrm{G}$ networks.

The contributions of this paper are as follows:

(i) The nonconvexity of the SCO-AN PA function makes it impossible to obtain the maximum value. In this paper, the SCO-AN PA function is reconstructed creatively using statistical theory, and the reconstructed PA function approximates the theoretical limit. From the reconstructed PA function, we can deduce the channel capacity of the legitimate channel and eavesdropping channel in large-scale MIMO. Thereby, the secrecy capacity in large-scale MIMO is deduced. We reach a crucial conclusion: the $\mathrm{PA}$ objective function of the channel between transmitter and receiver depends mainly on the partition of power and the number of transmitting antennas

(ii) Based on the reconstructed PA function, we derive the optimal PA coefficient. In large-scale MIMO, PA can be carried out according to this coefficient without calculation, and the system can achieve excellent security performance. To simplify the calculation, the precision of the traditional PA algorithm based on statistics is very low. We improve the traditional PA algorithm based on statistics and propose a new PA algorithm based on statistics. The accuracy of the PA algorithm is improved

(iii) We consider the effect of the imperfect CSI. An expression for ELB of SC with imperfect CSI is derived. Based on the expression, we conclude that we should generate more power for SCO-AN once the level of imperfect CSI increases. The negative effect of imperfect CSI on PA is proved by simulation

The rest of the paper is organized as follows: Section 2 introduces the related work. Section 3 defines the system, channel, and signal model. Section 4 investigates the ELB of SC. The optimal PA for the proposed system model is discussed in Section 5. The effect of the imperfect is studied in Section 6. Simulation results are given in Section 7. Finally, concluding remarks are given in Section 8.

\section{Related Work}

SCO-AN was first proposed in [16]. In this paper, the author proposes an SCO-AN to overcome the defects caused by the 
orthogonality of traditional artificial noise. For a long time, SCO-AN is not drawing attention. In recent years, the intelligent reflecting surface is a hot spot in the field of communication technology. IRS has two channels-uplink and downlink channels, and artificial noise cannot be orthogonal with two channels simultaneously. The nonorthogonal SCO$\mathrm{AN}$ is perfectly suitable for intelligent reflecting surface technology; it also brought new prospects for the application of SCO-AN. Hong et al. have started to add nonorthogonal artificial noise to the intelligent reflection surface and achieved excellent security performance [19].

Here, we briefly introduce the principle of AN. Let $\mathbf{H}$ be the CSI matrix. $\mathbf{H}$ is a $N_{h} \times N_{h}$ matrix. AN is a $N_{h} \times 1$ vector. $\mathrm{AN}$ is orthogonal to channel $\mathbf{H}$. AN should satisfy:

$$
\mathbf{H} \mathbf{w}_{k}=0 \text {, }
$$

$\mathbf{w}_{k}$ denotes AN. We find that AN can hardly be designed in the simulation. AN does not exist, especially when the number of transmitting antennas equals the number of receiving antennas. According to matrix theory, for a linear equation $\mathbf{H} \mathbf{w}_{k}=0$, if $\mathbf{H}$ is not full rank, that is, $|\mathbf{H}|=0, \mathbf{H w}_{k}$ $=0$ has a nonzero solution. However, in wireless communication, $|\mathbf{H}|=0$ almost does not exist. Therefore, for real-life communication, AN has only zero solutions, and AN cannot be designed.

SCO-AN is designed to solve the orthogonality defect of AN. SCO-AN is not orthogonal to channel $\mathbf{H}$; that is,

$$
\mathbf{H w}_{m}=\alpha,
$$

$\mathbf{w}_{m}$ denotes SCO-AN; $\alpha$ is a $N_{h} \times 1$ vector, and each elements in $\alpha$ is arbitrarily small. When designing SCO-AN, $\alpha$ satisfies condition $\operatorname{rank}(\mathbf{H})=\operatorname{rank}(\mathbf{H}, \alpha)$. According to matrix theory, SCO-AN must have a nonzero design scheme. The design of SCO-AN does not meet any restrictions.

We have performed a thousand simulation experiments to design AN and SCO-AN. From Table 1, it can be seen that when rank $(\mathbf{H})$ is equal to $N_{h}$, in 1000 experiments, $\mathrm{AN}$ is not designed successfully in any experiments. Only when rank $(\mathbf{H})$ is less than $N_{h}$, AN can be designed successfully. The design of SCO-AN is not affected by the $\operatorname{rank}(\mathbf{H})$.

Table 2 shows the number of solution vectors for $\mathrm{AN}$ and SCO-AN under different channel conditions, $N_{1}$ is the number of solution vectors for $\mathrm{AN}$, and $N_{2}$ is the number of solution vectors for SCO-AN. When $\operatorname{rank}(\mathbf{H})$ is equal to $N_{h}$, the number of solution vectors for $\mathrm{AN}$ is 0 , that is, $\mathrm{AN}$ cannot be designed, and the number of solution vectors of $\mathrm{SCO}-\mathrm{AN}$ is 1 , that is, under a specific power limit, there is only one design scheme of SCO-AN. When $\operatorname{rank}(\mathbf{H})-N_{h}$ $=1$, under a specific power limit, the number of AN solution vectors is 1; that is, under a specific power limit, there is only one design solution for AN. Moreover, there are countless kinds of SCO-AN design schemes. Only when $\operatorname{rank}(\mathbf{H})-$ $N_{h}>1$, the solution vectors of AN and SCO-AN are both infinite, which also shows that SCO-AN is a more designable than artificial noise.

A comparison of AN and SCO-AN secrecy performance is shown in Figure 1. The previous analysis shows that, in
TABLE 1: Design feasibility of AN and SCO-AN.

\begin{tabular}{lcc}
\hline & AN & SCO-AN \\
\hline $\operatorname{Rank}(\mathbf{H})=N_{h}$ & 0 & 1000 \\
$\operatorname{Rank}(\mathbf{H})<N_{h}$ & 1000 & 1000 \\
\hline
\end{tabular}

TABLE 2: Solution space size of AN and SCO-AN.

\begin{tabular}{lcc}
\hline & AN & SCO-AN \\
\hline $\operatorname{Rank}(\mathbf{H})=N_{h}$ & $N_{1}=0$ & $N_{2}=1$ \\
$\operatorname{Rank}(\mathbf{H})-N_{h}=1$ & $N_{1}=1$ & $N_{2}=\infty$ \\
$\operatorname{Rank}(\mathbf{H})-N_{h}>1$ & $N_{1}=\infty$ & $N_{2}=\infty$ \\
\hline
\end{tabular}

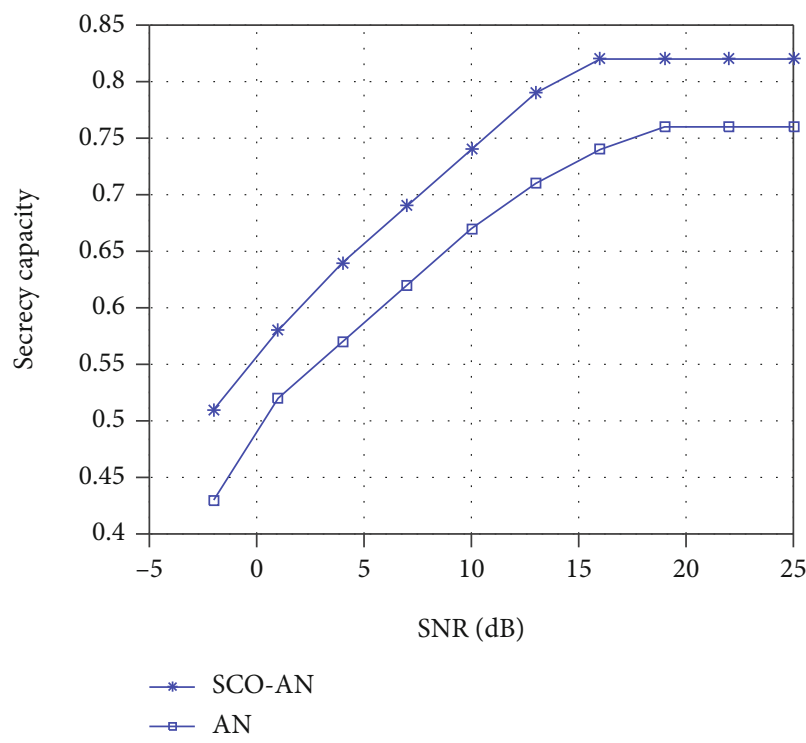

Figure 1: The SC of AN and SCO-AN versus different SNR.

most cases, the design of $\mathrm{AN}$ is very few or even unique, so for a particular channel, the improvement of AN for SC is limited; while the number of design scheme for SCO-AN is infinite, SCO-AN has no theoretical upper limit for the improvement of SC. Therefore, the SC of SCO-AN is always higher than that of AN.

In the real-life communication environment, Alice must consider the influence of power when transmitting signals. A reasonable power allocation will effectively increase the $\mathrm{SC}$ of the system. After considering the transmission power, the SC of the system is as follows:

$$
\begin{aligned}
C_{\mathrm{sec}}= & I\left(\mathbf{y}_{B} ; S\right)-I\left(\mathbf{y}_{E} ; S\right)=\log \left(1+\frac{\|\mathbf{H}\|^{2} \sigma_{u}^{2}}{\sigma_{n}^{2}+\|\mathbf{H}\|^{2}\left(P-\sigma_{u}^{2}\right)}\right) \\
& -\log \left(1+\frac{\|\mathbf{G}\|^{2} \sigma_{u}^{2}}{\|\mathbf{G}\|^{2}\left(P-\sigma_{u}^{2}\right)+\sigma_{e}^{2}}\right) .
\end{aligned}
$$

In (3), $\mathbf{H}$ and $\mathbf{G}$ are legitimate channel CSI and eavesdropping channel CSI, respectively. $\sigma_{n}^{2}$ and $\sigma_{e}^{2}$ are the variance 
of noise in legitimate channel and eavesdropping channel, respectively. $\sigma_{u}^{2}$ is the power of the transmitted signal, and the power of SCO-AN is $\sigma_{v}^{2} . \sigma_{u}^{2}+\sigma_{v}^{2}=P$; therefore, $\sigma_{v}^{2}=P$ $-\sigma_{u}^{2}$. If the variables in (3) are $\sigma_{v}^{2}$ and $\sigma_{u}^{2}$, the Hessian matrix of (3) is nonpositive definite so that (3) is nonconvex.

Due to the nonconvex property of the SCO-AN PA function, the extremum of the function and the optimal power allocation cannot be obtained. The iterative algorithms in [26-29] are applied to derive the maximum for PA function within a specific range. The iterative algorithms are affected by the initial value seriously and have a limited optimization effect.

Figure 2 shows a comparison of the different nonconvex optimization algorithms.

From Table 3, we can see that the different initial states of the transmitted signal and SCO-AN can lead to different optimization results, which is caused by the nonconvexity of the PA function. Therefore, traditional nonconvex optimization algorithms cannot get excellent optimization results. SQP is a classic iterative optimization algorithm. In the following sections, we continue to study the optimal PA and lower bound of secrecy capacity of SCO-AN under ideal conditions.

\section{Problem State}

3.1. System Model. In Figure 3, the system model of MIMO communication with eavesdroppers is shown. The noncolluding and colluding eavesdropper cases are discussed in this paper. Therefore, the system model has two scenarios: noncolluding and colluding eavesdroppers.

Alice is the transmitter of information. The number of Alice's antennas is $N_{K}$. Bob is the receiver of information. The number of Bob's antennas is $N_{P}$. Eve is the eavesdropper of information.

There are $N_{L}$ eavesdroppers in the system. For colluding scenario, the $N_{L}$ eavesdroppers can eavesdrop on information, which means that the eavesdropped information will be exchanged and processed jointly. The noncolluding eavesdropper is a special scenario that these $N_{L}$ eavesdroppers independently eavesdrop on information. When studying this scenario, we conducted research on each independent eavesdropper, which can be considered $N_{L}=1$. Similar settings can be found in $[15,30]$. For the facilitation of discussion, we assumed $N_{K}>N_{L}$ and $N_{K}=N_{P}$.

Here we have the following:

$$
\begin{aligned}
& \mathbf{y}_{B}=\mathbf{H} \mathbf{x}+\mathbf{n}, \\
& \mathbf{y}_{E}=\mathbf{G} \mathbf{x}+\mathbf{e},
\end{aligned}
$$

$\mathbf{y}_{B}$ is Bob's received signal, and $\mathbf{y}_{E}$ is Eve's eavesdropping signal. $\mathbf{H}$ is the CSI matrix of the legitimate channel. The dimension of $\mathbf{H}$ is $N_{K} \times N_{P}$. G is the CSI of eavesdropping channel. For the colluding scenario, $\mathbf{G}$ is a matrix and the dimension of $\mathbf{G}$ is $N_{K} \times N_{L}$; for the noncolluding scenario, $\mathbf{G}$ is a vector and the dimension of $\mathbf{G}$ is $N_{K} \times 1$. Each element in $\mathbf{H}$ and $\mathbf{G}$ is zero-mean complex Gaussian random variables and independent identically distributed (i.i.d). $\mathbf{x}$ is signal transmitted by Alice. $\mathbf{n}$ and $\mathbf{e}$ are the additive Gaussian

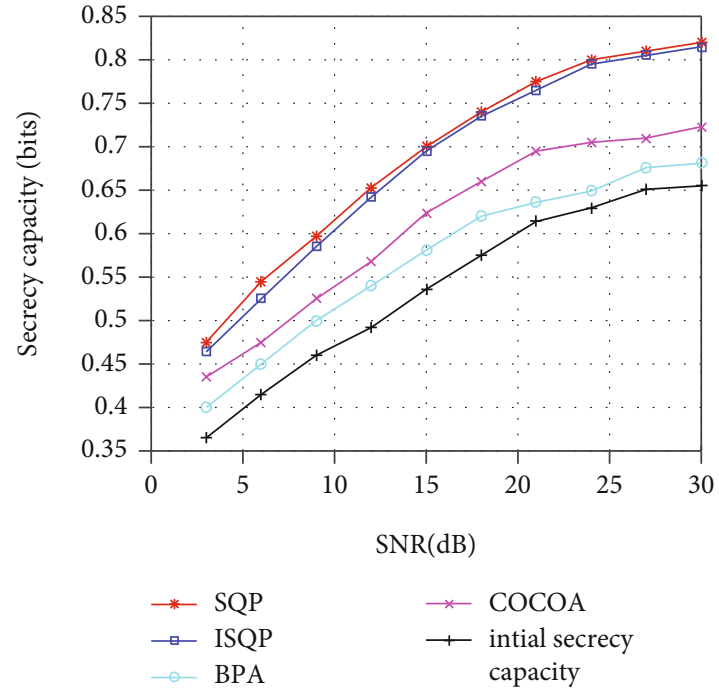

FIgURE 2: The comparison of the different nonconvex optimization algorithms.

TABLE 3: The initial state influence on SC.

\begin{tabular}{lccc}
\hline Algorithm & Initial $\left(\sigma_{u}^{2}, \sigma_{v}^{2}\right)$ & Initial SC & Optimized SC \\
\hline SQP & $(5,5)$ & 0.5612 & 0.6375 \\
SQP & $(3,7)$ & 0.4785 & 0.5364 \\
SQP & $(1,9)$ & 0.3821 & 0.4357 \\
\hline
\end{tabular}

white noise (AWGN) in legitimate and eavesdropping channels. The variance of $\mathbf{n}$ is $\sigma_{n}^{2}$. The variance of $\mathbf{e}$ is $\sigma_{e}^{2}$.

Because $\mathrm{AN}$ is orthogonal to $\mathbf{H}$, according to the theory of matrices, AN cannot be designed when $N_{K}=N_{P}$. The key idea of SCO-AN is to design a signal which does not show orthogonality to legitimate and eavesdropping channels. SCO-AN effectly reduces eavesdropping channel capacity while slightly reducing the capacity of legitimate channels [16]. Therefore, SCO-AN will improve the SC of the communication system.

We design the transmitted signal as below: $\mathbf{x}$ is a vector, the dimension of $\mathbf{x}$ is $N_{K} \times 1 . \mathbf{x}=\mathbf{r}_{1} \mathbf{u}+\mathbf{r}_{2} \mathbf{v}$, where $\mathbf{r}_{1}=\mathbf{H}^{\dagger} / \|$ $\mathbf{H} \| . \mathbf{u}$ is the signal which bears the information. The power of $\mathbf{u}$ is $\sigma_{u}^{2} . \mathbf{v}$ is the SCO-AN. The elements of $\mathbf{v}$ are i.i.d. In $\mathbf{v}$, each power of the $N_{K}-1$ column vectors is $\sigma_{v}^{2}$. [*] is the conjugate transpose operation of $* .\|*\|$ is the norm of *. So the legitimate receiving and eavesdropping signal becomes the following:

$$
\begin{aligned}
& \mathbf{y}_{B}=\mathbf{H r}_{1} \mathbf{u}+\mathbf{H r}_{2} \mathbf{v}+\mathbf{n}=\|\mathbf{H}\| \mathbf{u}+\mathbf{H}_{1} \mathbf{v}+\mathbf{n}, \\
& \mathbf{y}_{E}=\mathbf{G} \mathbf{r}_{1} \mathbf{u}+\mathbf{G} \mathbf{r}_{2} \mathbf{v}+\mathbf{e}=\mathbf{G}_{1} \mathbf{u}+\mathbf{G}_{2} \mathbf{v}+\mathbf{e},
\end{aligned}
$$

where $\mathbf{H}_{1}=\mathbf{H r}_{2}, \mathbf{G}_{1}=\mathbf{G r}_{1}$, and $\mathbf{G}_{2}=\mathbf{G r}_{2}$. 


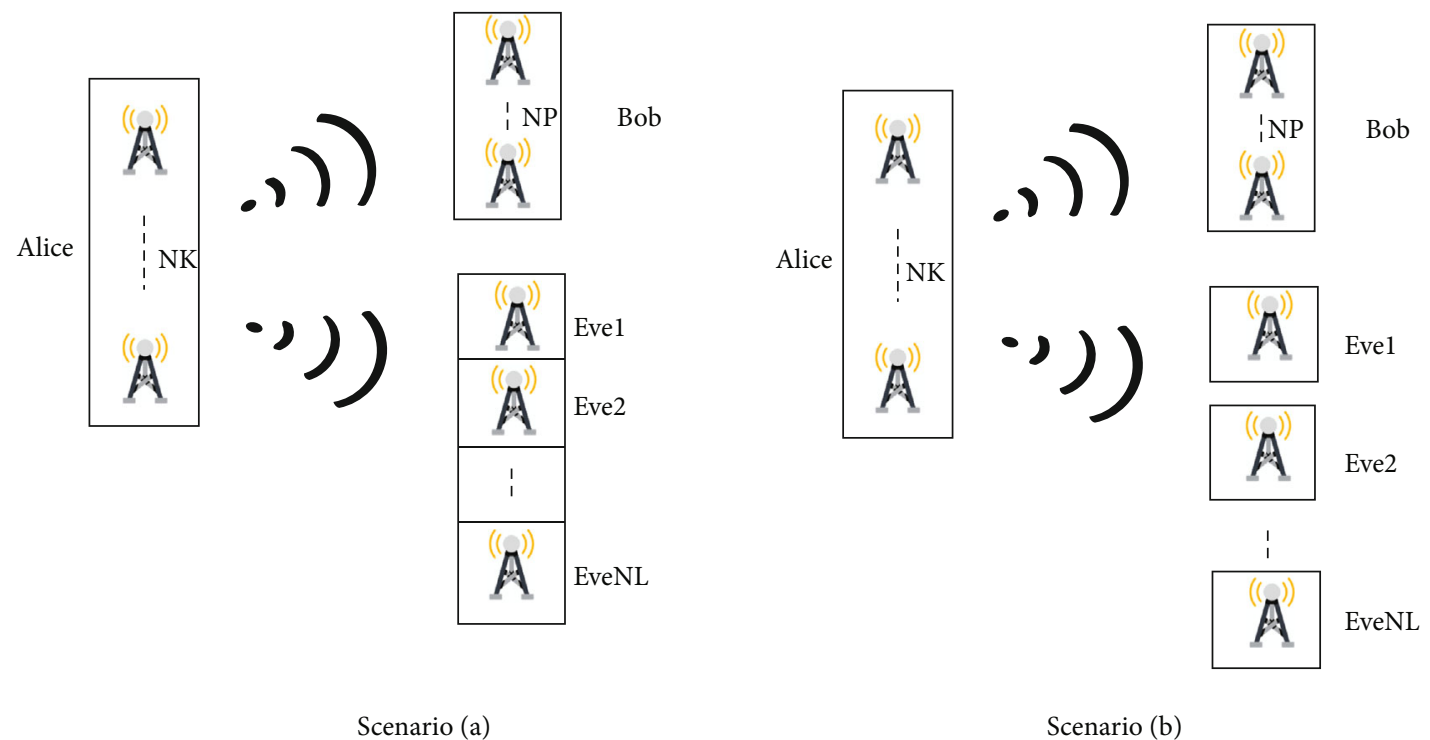

FIGURE 3: System model of MIMO communication with eavesdroppers.

Let the power for each transmission be $P, P=\sigma_{u}^{2}+$ $\left(N_{K}-1\right) \sigma_{v}^{2}$. The proportion of SCO-AN to $P$ is $\phi$. Here,

$$
\begin{aligned}
& \sigma_{u}^{2}=\phi P, \\
& \sigma_{v}^{2}=\frac{(1-\phi) P}{\left(N_{K}-1\right)} .
\end{aligned}
$$

From (8) and (9), we can come to the conclusion that Alice could adjust the PA strategy by adjusting $\phi$.

\section{ELB of SC for SCO-AN}

The ELB of SC for SCO-AN is derived in this section. The legitimate channel capacity is as follows:

$$
\begin{aligned}
C_{1} & =E_{H}\left\{\log _{2}\left(1+\frac{\sigma_{u}^{2}\|\mathbf{H}\|^{2}}{\sigma_{v}^{2}\|\mathbf{H}\|^{2}+\sigma_{n}^{2}}\right)\right\} \\
& =E_{H}\left\{\log _{2}\left(1+\frac{\sigma_{u}^{2}}{\left.\sigma_{v}^{2}+\sigma_{n}^{2} /\|\mathbf{H}\|^{2}\right)}\right\}\right. \\
& =E_{H}\left\{\log _{2}\left(1+\frac{\phi P}{(1-\phi) P / N_{K}-1+\sigma_{n}^{2} /\|\mathbf{H}\|^{2}}\right)\right\} \\
& =E_{H}\left\{\log _{2}\left(1+\frac{\phi}{1-\phi / N_{K}-1+\sigma_{n}^{2} /\|\mathbf{H}\|^{2} P}\right)\right\} .
\end{aligned}
$$

In (10), for the facilitation of discussion, we hypothesize the signal-noise-ratio (SNR) of communication is high enough, so $P \gg 1$. Therefore, the $\|\mathbf{H}\|^{2} P$ is much larger than $\sigma_{n}^{2}$ so $\sigma_{n}^{2} /\left(\|\mathbf{H}\|^{2} P\right)$ is an arbitrarily small number which can be ignored. (10) is written as follows:
$C_{1}=\log _{2}\left(1+\frac{\phi P}{(1-\phi) P / N_{K}-1}\right)=\log _{2}\left(1+\frac{\phi}{1-\phi / N_{K}-1}\right)$.

From (11), we can conclude that the capacity of the legitimate channel is mainly related to $N_{k}$ and $\phi$ in a high SNR regime. Alice can adjust the capacity of the legitimate channel by adjusting $\phi$ and $N_{K}$.

Alice has no knowledge of SNR of G. In order to enable secure communication, we assume that $\sigma_{e}^{2}$ is arbitrarily tiny, which means the eavesdropper has the best communication environment.

Therefore, the upper bound on the eavesdropping channel capacity without considering $\sigma_{e}^{2}$ is as follows:

$$
\begin{aligned}
C_{2} & =E_{h, \mathbf{G}_{1}, \mathbf{G}_{2}}\left\{\log _{2}\left|I+\sigma_{u}^{2} \mathbf{G}_{1} \mathbf{G}_{1}^{\dagger}\left(\sigma_{v}^{2} \mathbf{G}_{2} \mathbf{G}_{2}^{\dagger}\right)^{-1}\right|\right\} \\
& =E_{h, \mathbf{G}_{1}, \mathbf{G}_{2}}\left\{\log _{2}\left(1+\frac{N_{K}-1}{r-1} \mathbf{G}_{1}^{\dagger}\left(\mathbf{G}_{2} \mathbf{G}_{2}^{\dagger}\right)^{-1} \mathbf{G}_{1}\right\} .\right.
\end{aligned}
$$

$r$ is the inverse of $\phi, r=\phi^{-1}$.

$\mathbf{R}=\left[\mathbf{r}_{1} \mathbf{r}_{2}\right]$ is a unitary matrix. $\mathbf{G R}=\left[\mathbf{G}_{1} \mathbf{G}_{2}\right]$ is a complex Gaussian matrix, and $\mathbf{G}_{1}$ and $\mathbf{G}_{2}$ are independent. Therefore, $\mathbf{G}_{1}^{\dagger}\left(\mathbf{G}_{2} \mathbf{G}_{2}^{\dagger}\right) \mathbf{G}_{1}$ is equivalent to the signal-to-interference ratio of a $N_{L}$-branch mininum mean square error (MMSE) diversity combiner with $N_{K}-1$ interferers. According to $[30,31]$, the complementary cumulative distribution function of $X=\mathbf{G}_{1}^{\dagger}\left(\mathbf{G}_{2} \mathbf{G}_{2}^{\dagger}\right)^{-1} \mathbf{G}_{1}$ is as follows:

$$
M_{X}(x)=\frac{\sum_{n=0}^{N_{L}-1}\left(\begin{array}{c}
N_{K}-1 \\
n
\end{array}\right) x^{n}}{(1+x)^{N_{K}-1}} .
$$


According to (13), we can rewrite (12) as follows:

$$
\begin{aligned}
C_{2}= & E_{h}\left\{\frac{1}{\ln 2} \int_{0}^{\infty} \frac{N_{K}-1}{r-1}\left(1+\frac{N_{K}-1}{r-1} x\right)^{-1} M_{x}(x) d x\right\} \\
= & E_{h}\left\{\frac{1}{\ln 2} \sum_{n=0}^{N_{L}-1}\left(\begin{array}{c}
N_{K}-1 \\
k
\end{array}\right) \frac{N_{K}-1}{r-1} \operatorname{Bet}\left(k+1, N_{K}-1-k\right)\right. \\
& \left.\times K_{1}\left(1, k+1 ; N_{K} ; \frac{r-N_{K}}{r-1}\right)\right\} .
\end{aligned}
$$

In (14), $\operatorname{Bet}(\cdot)$ is the function of beta distribution and $\operatorname{Bet}(a, b)=\Gamma(a) \Gamma(b) / \Gamma(a+b) . K_{1}(\cdot)$ is Gaussian hypergeometric function. Formula (14) uses conclusion in [31].

From [5], the ELB of SC is $C=\left[C_{1}-C_{2}\right]^{+}$and $[a]^{+}$ denotes $\max \{0, \alpha\}$. The ELB of SC is as follows:

$$
\begin{aligned}
C= & \frac{1}{\ln 2}\left[\left\{\log _{2}\left(1+\frac{N_{K}-1}{(r-1)}\right)-\sum_{n=0}^{N_{L}-1}\left(\begin{array}{c}
N_{K}-1 \\
k
\end{array}\right) \frac{N_{K}-1}{r-1}\right.\right. \\
& \left.\left.\times \operatorname{Bet}\left(k+1, N_{K}-1-k\right) \times K_{1}\left(1, k+1 ; N_{K} ; \frac{r-N_{K}}{r-1}\right)\right\}\right]^{+} .
\end{aligned}
$$

In the following sections, the simplified and approximated expressions on ELB of SC will be discussed.
In (15), from [32], here we have

$$
\begin{aligned}
K_{1}(1,1 ; M+1 ; y) & =(1+y)^{M-1} K_{1}(M, M ; M+1 ; y), \\
\frac{d^{M-1}}{d y^{M-1}} K_{1}(1,1 ; 2 ; y) & =\frac{(1)_{M-1}(1)_{M-1}}{(2)_{M-1}} K_{1}(M, M ; M+1 ; y),
\end{aligned}
$$

where $(m)_{n}$ is the rising factorial. So,

$$
\begin{array}{r}
K_{1}(M, M ; M+1 ; y)=\frac{(2)_{M-1}}{(1)_{M-1}(1)_{M-1}} \frac{d^{M-1}}{d y^{M-1}} K_{1}(1,1 ; 2 ; y) \\
=-\frac{M}{(M-1) !} \sum_{t=0}^{M-1}\left(\begin{array}{c}
M-1 \\
t
\end{array}\right) \frac{d^{t}}{d y^{t}} \ln (1-y) \frac{d^{M-1-t}}{d y^{M-1-t}} y^{-1} .
\end{array}
$$

In [32], we know $K_{1}(1,1 ; 2 ; y)=-\ln (1-y) / y$. Therefore, here, we have

$$
\begin{aligned}
\frac{d^{t}}{d y^{t}} \ln (1-y) & =-\frac{(t-1) !}{(1-y)^{t}}, t=1,2,3 \cdots, \\
\frac{d^{t}}{d y^{t}} y^{-1} & =\frac{(-1)^{t} t !}{y^{t+1}}, t=0,1,2,3 \cdots
\end{aligned}
$$

Substituting (19) into (18), we can obtain the following:

$$
\begin{aligned}
K_{1}(M, M ; M+1 ; y) & =-\frac{M}{(M-1) !} \times\left(\ln (1-y) \frac{(-1)^{M-1}(M-1) !}{y^{M}}-\sum_{t=1}^{M-1} \frac{(M-1) !}{t !(M-1-t) !} \frac{(t-1) !}{(1-y)^{t}} \frac{(-1)^{M-1-t}(M-1-t) !}{y^{M-t}}\right) \\
& =\frac{(-1)^{M} M}{y^{M}}\left(\ln (1-y)-\sum_{t=1}^{M-1} \frac{1}{t} \frac{y^{t}}{(y-1)^{t}}\right) .
\end{aligned}
$$

From (20), it is easy to obtain

$K_{1}(1,1 ; M+1 ; y)=\frac{(-1)^{M} M(1-y)^{M-1}}{y^{M}}\left(\ln (1-y)-\sum_{t=1}^{M-1} \frac{1}{t} \frac{y^{t}}{(y-1)^{t}}\right)$.

4.1. The Noncolluding Eavesdropper Case for SC. In the noncolluding eavesdroppers case, there is no cooperation between each eavesdropper; therefore, $N_{L}=1$. Then,

$$
\begin{aligned}
C_{2} & =E_{h}\left\{\frac{1}{\ln 2} \times \frac{1}{r-1} K_{1}\left(1,1 ; N_{K} ; \frac{r-N_{K}}{r-1}\right)\right\} \\
& =E_{h}\left\{\frac{1}{\ln 2}\left(\frac{N_{K}-1}{N_{K}-r}\right)^{N_{K}-1} \times\left(\ln \left(\frac{N_{K}-1}{r-1}\right)-\sum_{l=1}^{N_{K}-2}\left(\frac{N_{K}-r}{N_{K}-1}\right)^{l}\right)\right\} .
\end{aligned}
$$

(22) is derived by applying the Gaussian hypergeometric function. The ELB of SC for noncolluding eavesdroppers is given by the following:

$$
\begin{aligned}
C= & \frac{1}{\ln 2}\left\{\left[\left\{\log _{2}\left(1+\frac{N_{K}-1}{(r-1)}\right)\right\}-E_{h}\left\{\left(\frac{N_{K}-1}{N_{K}-r}\right)^{N_{K}-1}\right.\right.\right. \\
& \left.\left.\left.\times\left(\ln \left(\frac{N_{K}-1}{r-1}\right)-\sum_{l=1}^{N_{K}-2}\left(\frac{N_{K}-r}{N_{K}-1}\right)\right)\right\}\right]^{+}\right\}
\end{aligned}
$$

4.2. The Colluding Eavesdropper Case for SC. In this subsection, the ELB of SC is discussed when $N_{K}$ is large enough. 
We know that $\lim _{N_{K} \longrightarrow \infty} \mathbf{G}_{2} \mathbf{G}_{2}^{\dagger} /\left(N_{K}-1\right)=I$ when we use the strong law of large numbers. So (12) can be written as follows:

$$
\begin{aligned}
\lim _{N_{K} \longrightarrow \infty} C_{2} & =\lim _{N_{K} \longrightarrow \infty} E_{\mathbf{G}_{1}, \mathbf{G}_{2}}\left\{\log _{2}\left(1+\frac{1}{r-1} \mathbf{G}_{1}^{\dagger}\left(\frac{\mathbf{G}_{2} \mathbf{G}_{2}^{\dagger}}{N_{K}-1}\right)^{-1} \mathbf{G}_{1}\right)\right\} \\
& =E_{\mathbf{G}_{1}}\left\{\log _{2}\left(1+\frac{1}{r-1}\left\|\mathbf{G}_{1}\right\|^{2}\right)\right\} \\
& =\frac{1}{\ln 2} \exp (r-1) \sum_{n=1}^{N_{L}} E_{k}(r-1),
\end{aligned}
$$

where $E_{k}()$ is the generalized exponential integral. The channel response of Rayleigh fading channel is an exponential function. So $\left\|G_{1}\right\|^{2}$ is a Gamma function. The paramater of $\left\|\mathbf{G}_{1}\right\|^{2}$ is $\left(N_{L}, 1\right)$.

When $N_{K}$ is large enough, the ELB of SC is

$$
C=\left[\log _{2}\left(1+\frac{N_{K}-1}{(r-1)}\right)-\frac{1}{\ln 2} \exp (r-1) \sum_{n=1}^{N_{L}} E_{k}(r-1)\right]^{+}
$$

From (25), we can conclude that the ELB of SC relates to $N_{k}$ and $r$. The optimal PA can be obtained by deriving the ELB of SC. The optimal PA is a function of $N_{k}$ and $r$.

\section{Optimal PA of SCO-AN}

The ELB of SC is discussed in the last section. In this section, the optimal PA of the SCO-AN system is studied. The ELB of SC for noncolluding eavesdroppers and infinite $N_{K}$ are obtained by (23) and (25). For the facilitation of calculation and discussion, the expressions obtained in the last sections will be applied in this section.

5.1. Optimal PA: Noncolluding Eavesdropper. For the noncolluding case, the $N_{L}=1$, and the ELB of SC $C=\left[C_{1}-C_{2}\right]^{+}$in last sections helps us to find the optimal values for the PA coefficients $r$ and $\phi$.

The first derivatives of (23) - the ELB of SC-concerning the PA coefficient $r$ will help us to find the optimal PA coefficient. Therefore, the first derivative of (23) satisfies the following:

$$
\frac{d C}{d r}=\frac{d C_{1}}{d r}-\frac{d C_{2}}{d r}=0
$$

From (26), the optimal PA coefficient should satisfy

$$
\begin{aligned}
& {\left[\frac{1}{\ln 2\left[(r-1)^{2}+\left(N_{K}-1\right)(r-1)\right]}-\left\{\left(N_{K}-1\right) \times\left(\frac{N_{K}-1}{N_{K}-r}\right)^{N_{K}-2}\right.\right.} \\
& \quad \times \frac{1-N_{K}}{\left(N_{K}-r\right)^{2}} \times\left(\ln \left(\frac{N_{K}-1}{r-1}\right)-\frac{N_{K}-r}{N_{K}-1}\right)+\left(\frac{N_{K}-1}{N_{K}-r}\right)^{N_{K}-1} \\
& \left.\quad \times\left(\frac{r-1}{N_{K}-1} \times \frac{1-N_{K}}{(r-1)^{2}}+\frac{1}{N_{K}-1}\right)\right]^{+}=0 .
\end{aligned}
$$

(27) is a higher-order equation of $r$ on $N_{K}$. Its closed-form solution is challenging to find or even nonexistent. Moreover, according to the difference of $N_{K}$, its root-finding formula is also different. Therefore, in the following research content, we provide some of the optimal PA coefficients with a particular number of commonly used antennas.

5.2. Optimal PA: Colluding Eavesdroppers. In this subsection, the colluding eavesdropper's case is studied. We improve the optimal PA coefficient algorithm under colluding eavesdroppers in the classic paper [30].

In [30], for the convenience of calculation, $\exp (r-1)$ $\sum_{n=1}^{N_{L}} E_{k}(r-1)$ is simplified to $\exp (r-1) E_{k}(r-1)$. The first $N_{L}-1$ items in $\exp (r-1) \sum_{n=1}^{N_{L}} E_{k}(r-1)$ have been omitted. This will cause large errors in the calculation results. So we will improve this formula in order to get better calculation results.

Considering the expression of $C$ in (25) and taking its derivative concerning $r$, the optimal $r$ satisfies the following:

$$
\begin{aligned}
\frac{d C}{d r}= & \frac{d C_{1}}{d r}-\frac{d C_{2}}{d r}=\left(\ln 2\left(r-N_{K}-2\right)\right)^{-1} \\
& -(\ln 2(r-1))^{-1}-e^{r-1}\left(\frac{N_{L}\left(N_{L}-1+2(r-1)\right)}{2(r-1)}\right) \sum_{n=1}^{N_{L}} E_{k}(r-1)-\frac{N_{L}}{r-1} .
\end{aligned}
$$

Hence, we have shown that for sufficiently large $N_{K}$, the optimal PA is given by (28). The solution of Equation (28) is the optimal PA coefficient. The optimal ratio of PA depends on $N_{L}$ and $N_{K}$.

In Table 4, we provide the optimal PA coefficients for some commonly used transmitting and eavesdropping antennas. Alice can allocate power to the transmitted signal by looking up the table instead of using an algorithm for power allocation. From Table 4, we can also see that when the number of eavesdropping antennas increases, it is wise to allocate more power to the SCO-AN to confuse the eavesdropper. At the same time, when $N_{K}$ increases, the capacity of the legitimate channel increases, which can reduce the power allocated for SCO-AN.

\section{Secrecy Capacity: Imperfect Channel State Information}

The previous section explored the ELB of SC obtained with the perfect CSI. We will continue to discuss the effect of the imperfect CSI. 
TABle 4: Optimal $\phi$ for different $N_{K}$ and $N_{L}$.

\begin{tabular}{|c|c|c|}
\hline Number of $N_{K}$ & Number of $N_{L}$ & Optimal $\phi$ \\
\hline 2 & 1 & 0.292 \\
\hline 2 & 2 & 0.313 \\
\hline 2 & 4 & 0.327 \\
\hline 2 & 8 & 0.339 \\
\hline 4 & 1 & 0.271 \\
\hline 4 & 2 & 0.282 \\
\hline 4 & 4 & 0.312 \\
\hline 4 & 8 & 0.327 \\
\hline 8 & 1 & 0.255 \\
\hline 8 & 2 & 0.262 \\
\hline 8 & 4 & 0.272 \\
\hline 8 & 8 & 0.284 \\
\hline 16 & 1 & 0.232 \\
\hline 16 & 2 & 0.238 \\
\hline 16 & 4 & 0.252 \\
\hline 16 & 8 & 0.267 \\
\hline 32 & 1 & 0.207 \\
\hline 32 & 2 & 0.212 \\
\hline 32 & 4 & 0.232 \\
\hline 32 & 8 & 0.242 \\
\hline 64 & 1 & 0.185 \\
\hline 64 & 2 & 0.202 \\
\hline 64 & 4 & 0.231 \\
\hline 64 & 8 & 0.252 \\
\hline 128 & 1 & 0.171 \\
\hline 128 & 2 & 0.190 \\
\hline 128 & 4 & 0.201 \\
\hline 128 & 8 & 0.224 \\
\hline
\end{tabular}

The CSI estimation error introduces extra noise into the legitimate channel. Bob uses the channel estimation algorithm to get the CSI. The CSI is imperfect due to the Gaussian noise. Therefore,

$$
\mathbf{H}=\widehat{\mathbf{H}}+\tilde{\mathbf{H}},
$$

where $\widehat{\mathbf{H}}$ denotes the channel state information obtained by MMSE channel estimate and $\tilde{\mathbf{H}}$ denotes the channel estimation error. $\widehat{\mathbf{H}}$ and $\tilde{\mathbf{H}}$ are uncorrelated due to the property of the MMSE estimation. Therefore,

$$
\sigma_{H}^{2}=\sigma_{\widehat{H}}^{2}+\sigma_{\tilde{H}}^{2}
$$

$\mathbf{r}_{1}$ is designed as $\mathbf{r}_{1}=\mathbf{H} \wedge^{\dagger} /\|\widehat{\mathbf{H}}\|$, and the symbol received by Bob is

$$
\mathbf{y}_{B}=\widehat{\mathbf{H}} \mathbf{x}+\tilde{\mathbf{H}} \mathbf{x}+\mathbf{H r}_{2} \mathbf{v}+n=\|\widehat{\mathbf{H}}\| \mathbf{u}+\tilde{\mathbf{H}} \mathbf{R}\left[\mathbf{u v}^{T}\right]^{T}+\mathbf{H}_{1} \mathbf{v}+n,
$$

where $\mathbf{H}_{1}=\mathbf{H r}_{2}$. $\mathbf{R}$ is a unitary matrix, so the distribution of $\tilde{\mathbf{h}} \mathbf{R}$ and $\tilde{\mathbf{h}}$ is the same. Therefore, the ELB of SC for the legitimate channel is

$$
\widehat{C}_{1}=E_{h}\left\{\log _{2}\left(1+\frac{\sigma_{u}^{2}\|\mathbf{H} \wedge\|^{2}}{\sigma_{v}^{2}\|\mathbf{H} \wedge\|^{2}+\sigma_{\tilde{H}}^{2} P+1}\right)\right\} .
$$

With $\sigma_{H}^{2}$ normalized to unity, we have $\sigma_{\widehat{H}}^{2}+\sigma_{\tilde{H}}^{2}=1$. Each subchannel between Alice and Bod obeys exponential distribution. Therefore, $\|\mathbf{H} \wedge\|^{2}$ follows a Gamma distribution with parameter $\left(N_{K}, 1-\sigma_{\tilde{H}}^{2}\right)$. Here, we have

$$
\widehat{C}_{1}=\int_{0}^{\infty} g(x) \frac{\left(1-\sigma_{\tilde{H}}^{2}\right)^{N_{K}-1}}{\Gamma\left(N_{K}\right)}[H(x)]^{N_{K}-1} e^{-\left(1-\sigma_{\tilde{H}}^{2}\right) h(x)} d x .
$$

In (33), $h(x)$ is the inverse function $\left(\|\mathbf{H} \wedge\|^{2}=x\right)$ of (32), and $g(x)$ is the first derivative of $h(x)$. Therefore,

$$
\begin{aligned}
& h(x)=\ln 2\left\{\frac{2^{x}\left(2^{x}-1\right)\left(1+\sigma_{\widehat{H}}^{2} P\right) \sigma_{v}^{2}}{\left(\sigma_{u}^{2}-\left(2^{x}-1\right) \sigma_{v}^{2}\right)^{2}}+\frac{2^{x}\left(1+\sigma_{\widehat{H}}^{2} P\right)}{\sigma_{u}^{2}-\left(2^{x}-1\right) \sigma_{v}^{2}}\right\} \\
& g(x)=\frac{\left(2^{x}-1\right)\left(1+\sigma_{\widehat{H}}^{2} P\right)}{\sigma_{u}^{2}-\left(2^{x}-1\right) \sigma_{v}^{2}}
\end{aligned}
$$

The error of channel estimation does not affect Eve's channel. Therefore, the ELB of SC is as follows:

$$
\begin{aligned}
C= & {\left[\int_{0}^{\infty} h(x) \frac{\left(1-\sigma_{\tilde{H}}^{2}\right)^{N_{K}-1}}{\Gamma\left(N_{K}\right)}[g(x)]^{N_{K}-1} e^{-\left(1-\sigma_{\tilde{H}}^{2}\right) g(x)} d x\right.} \\
& \left.-\frac{1}{\ln 2}\left(\psi^{(0)}\left(N_{L}+r\right)-\psi^{(0)}(r)\right)\right]^{+},
\end{aligned}
$$

where $\Gamma()$ denotes the Gamma function and $\Psi^{(n)}()$ denotes the $n_{\text {th }}$ derivative of the polygamma function.

\section{Results and Discussion}

7.1. Simulation Parameters. In the simulation, the legitimate and eavesdropping channels are Rayleigh fading channels. The relevant simulation parameters are shown in Table 5.

7.2. Simulation Results and Discussion. Figure 4 shows how ELB of SC changes with $N_{k}$ and $N_{l}$. The ELB of SC is calculated by (15) with the optimal $\phi$. It can be seen from Figure 4 that when the number of transmitting antennas $N_{k}$ increases, the SC also increases. It can also be seen from (15) that $N_{k}$ is located on the molecule, and $N_{k}$ plays a positive role in the promotion of SC. Similarly, the increase in the number of eavesdropping antennas will reduce the SC of the system. The above conclusions are easy to understand. The increase in the number of transmitting antennas represents an increase in the capacity of the legitimate channel. Therefore, 
TABLE 5: Simulation environment.

\begin{tabular}{lc}
\hline Parameters & Values \\
\hline$N_{k}$ & From 1 to 256 \\
$N_{L}$ & From 1 to 256 \\
Power for each transmission & $10(\mathrm{~W})$ \\
Mean of legitimate and eavesdropping channels & 0 \\
Variances of legitimate and eavesdropping channels & 0.5 \\
SNR of legitimate and eavesdropping channels & From -5 to 30 (dB)
\end{tabular}

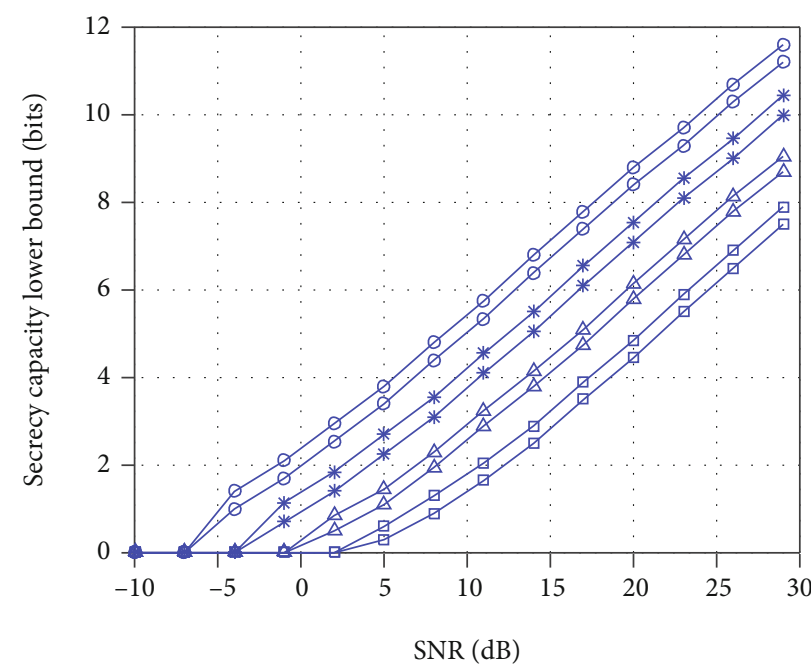

$$
\begin{array}{ll}
\rightarrow \mathrm{N}_{\mathrm{K}}=8, \mathrm{~N}_{\mathrm{L}}=1, \mathrm{AN} & \rightarrow \mathrm{N}_{\mathrm{K}}=8, \mathrm{~N}_{\mathrm{L}}=1, \text { SCO-AN } \\
\rightarrow \mathrm{N}_{\mathrm{K}}=4, \mathrm{~N}_{\mathrm{L}}=1, \mathrm{AN} & \rightarrow-\mathrm{N}_{\mathrm{K}}=4, \mathrm{~N}_{\mathrm{L}}=1, \text { SCO-AN } \\
\square \mathrm{N}_{\mathrm{K}}=4, \mathrm{~N}_{\mathrm{L}}=2, \mathrm{AN} & \rightarrow \mathrm{N}_{\mathrm{K}}=4, \mathrm{~N}_{\mathrm{L}}=2 \text {, SCO-AN } \\
\rightarrow \mathrm{N}_{\mathrm{K}}=8, \mathrm{~N}_{\mathrm{L}}=6, \mathrm{AN} & \rightarrow-\mathrm{N}_{\mathrm{K}}=8, \mathrm{~N}_{\mathrm{L}}=6, \text { SCO-AN }
\end{array}
$$

Figure 4: The ELB of SC versus SNR with different $N_{K}$ and $N_{L}$.

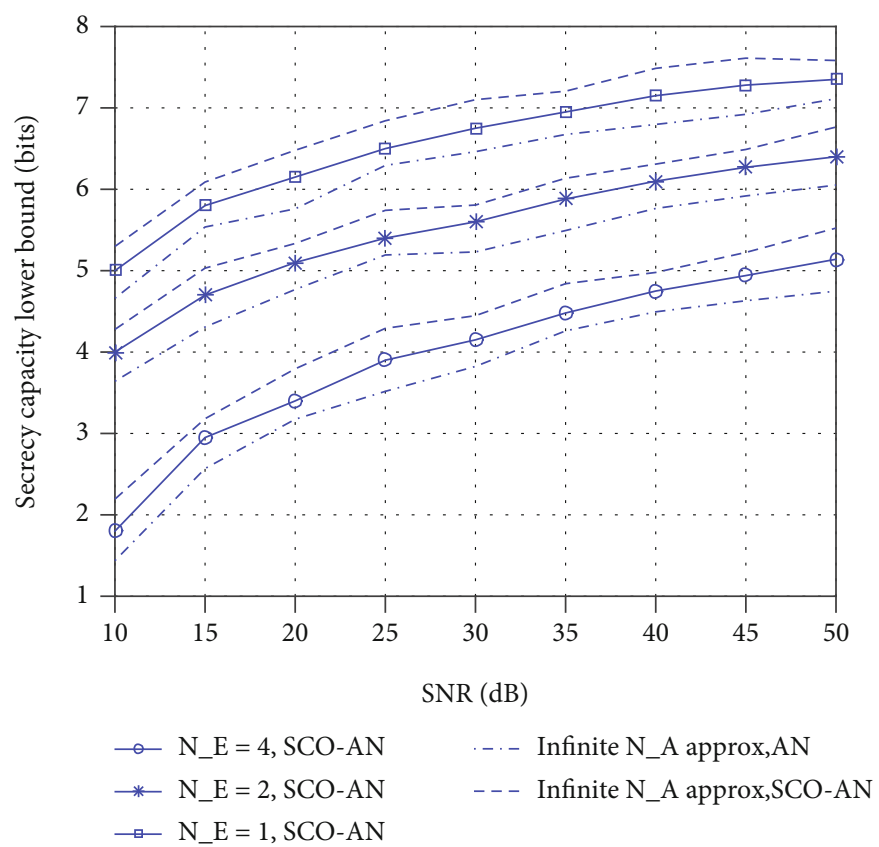

Figure 5: The lower bound of SC versus SNR with different numbers of $N_{L}$ and infinite $N_{K}$. 


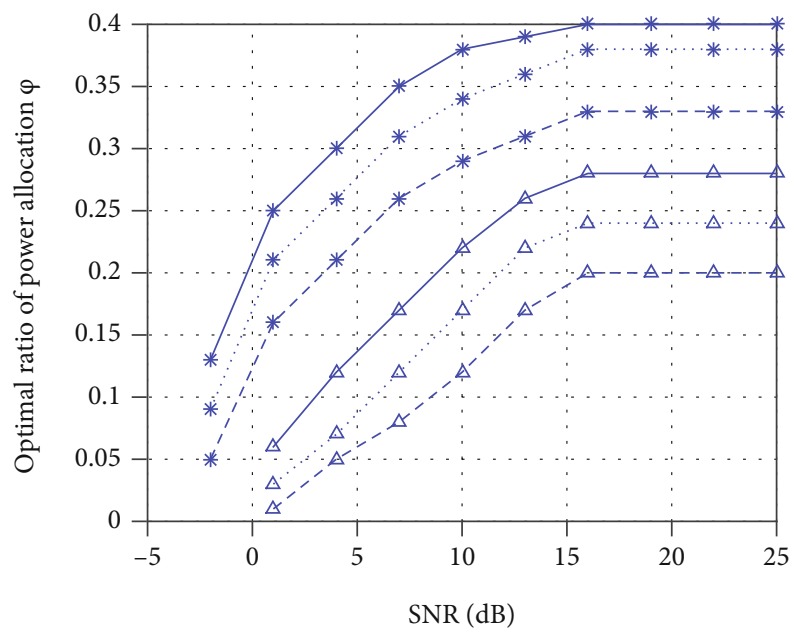

$$
\begin{aligned}
*-\mathrm{N}_{\mathrm{K}}=64, \text { error variance }=0.15 \\
\cdots * . \mathrm{N}_{\mathrm{K}}=64, \text { error variance }=0.05 \\
-*-\mathrm{N}_{\mathrm{K}}=64, \text { error variance }=0 \\
\triangle-\mathrm{N}_{\mathrm{K}}=32, \text { error variance }=0 \\
\therefore . \mathrm{N}_{\mathrm{K}}=32, \text { error variance }=0.05 \\
-\triangle-\mathrm{N}_{\mathrm{K}}=32, \text { error variance }=0.15
\end{aligned}
$$

Figure 6: The lower bound of SC versus ratio of power $\phi$ with different $N_{K}$.

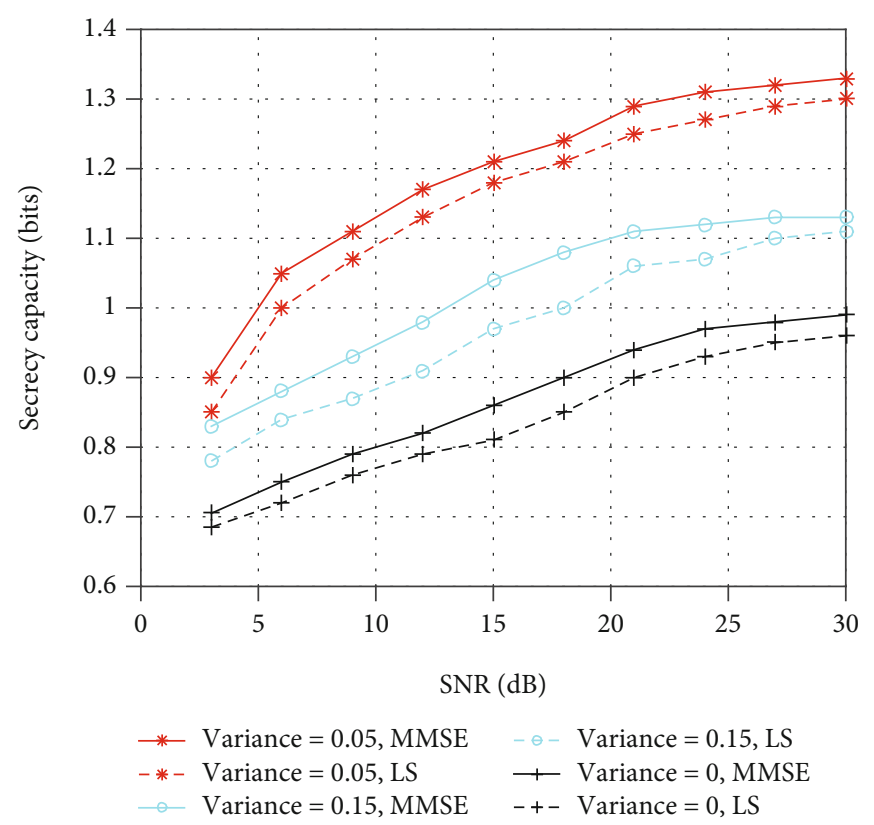

FIgURE 7: $\phi$ versus SNR for different $N_{K}$ and channel estimation errors $\sigma_{\tilde{H}}^{2}$.

the SC will increase. Similarly, the increase in the number of eavesdropping antennas will result in a decrease in the SC.

Figure 5 shows the effect of the number of eavesdropping antennas on the SC. It can be seen that with the increase in the number of eavesdropping antennas, the SC of the wireless communication system decreases under the condition that the legitimate channel remains unchanged. At the same time, Figure 5 shows the SC of the wireless communication system for $\mathrm{AN}$ and SCO-AN when $\mathrm{N}$ approaches infinity. It can be seen that the ELB on SC of SCO-AN is higher than that of AN.
Figure 6 shows the ELB of SC in (15) with the optimal $\phi$. For comparison, the ELB of SC for AN with equal power allocation is indicated by solid lines. ELB of SC for SCO-AN is always greater than that for AN. The SC increases as $N_{K}$ grows. The optimal $\phi$ of SCO-AN is much smaller than that of $\mathrm{SC}$ for $\mathrm{AN}$, which means SCO-AN has a better secrecy performance with less power for extra artificial noise. It proves that SCO-AN is a better artificial noise when compared to $\mathrm{AN}$. The SC of the wireless communication system decreases, which shows that the channel estimation error has a 


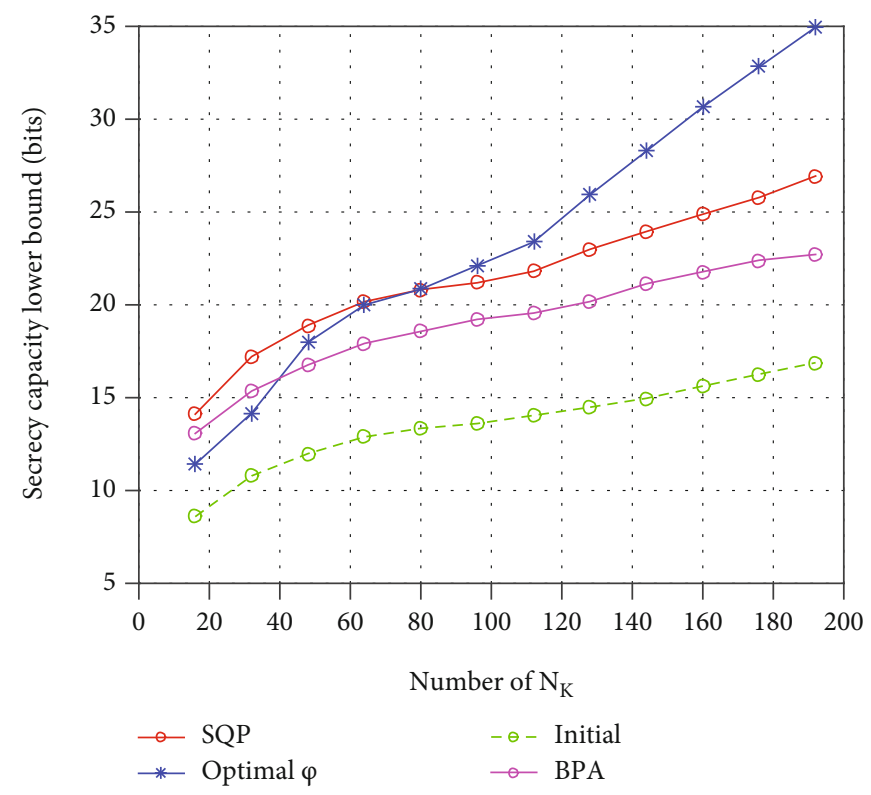

Figure 8: The ELB of SC versus $N_{K}$ with different PA algorithm.

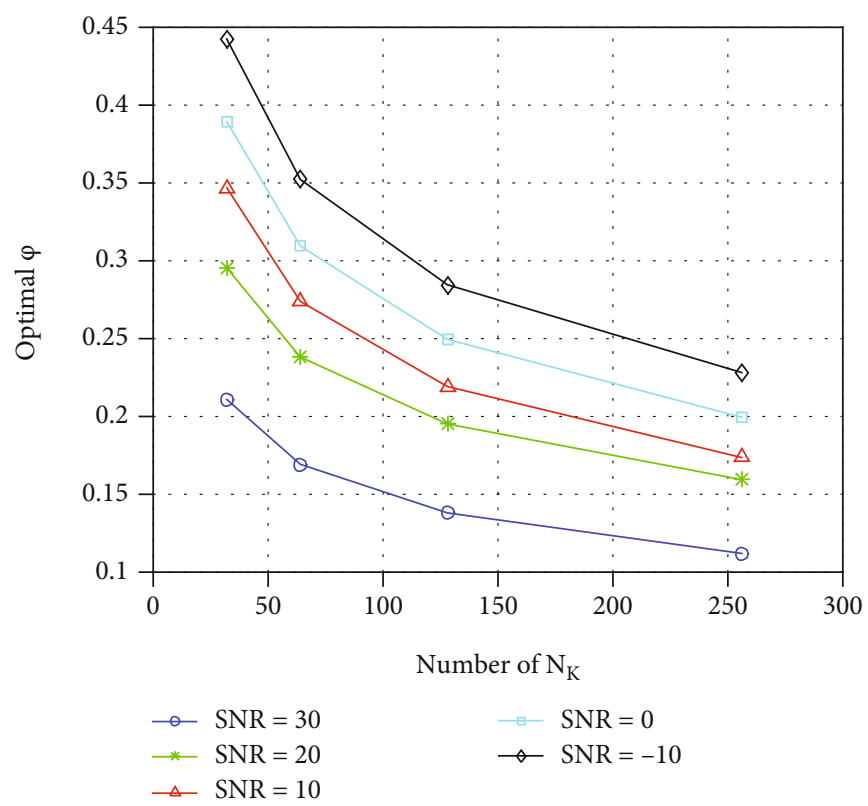

FIgURE 9: The optimal power distribution coefficient versus SNR with different $N_{K}$.

counterproductive effect on the security performance of the wireless communication system, which is also in line with reality. The imperfect CSI will cause Alice to error in the distribution of transmitting power, which will leads to a decrease in the SC.

The AWGN produces the CSI estimation error. So the CSI estimation error decreases with SNR. The deviation of the PA ratio between the practical system and the perfect CSI system becomes smaller with the increase of SNR. We should consider the channel estimation error when SNR is low in a practical system.
Figure 7 shows the SC with optimal $\phi$ and different types of $\sigma_{\tilde{H}}^{2}$ (LS and MMSE) [31]. MMSE and LS are classic channel estimation algorithms. It can be seen that for the influence of different levels of imperfect CSI on SC, the influence of wireless communication systems using MMSE channel estimation is always less than that of LS, which is because the MMSE channel estimation accuracy is higher than that of the LS, so the MMSE channel estimation has less influence on Alice's power allocation. At the same time, the increase of the channel estimation error level will cause the decrease of SC. 


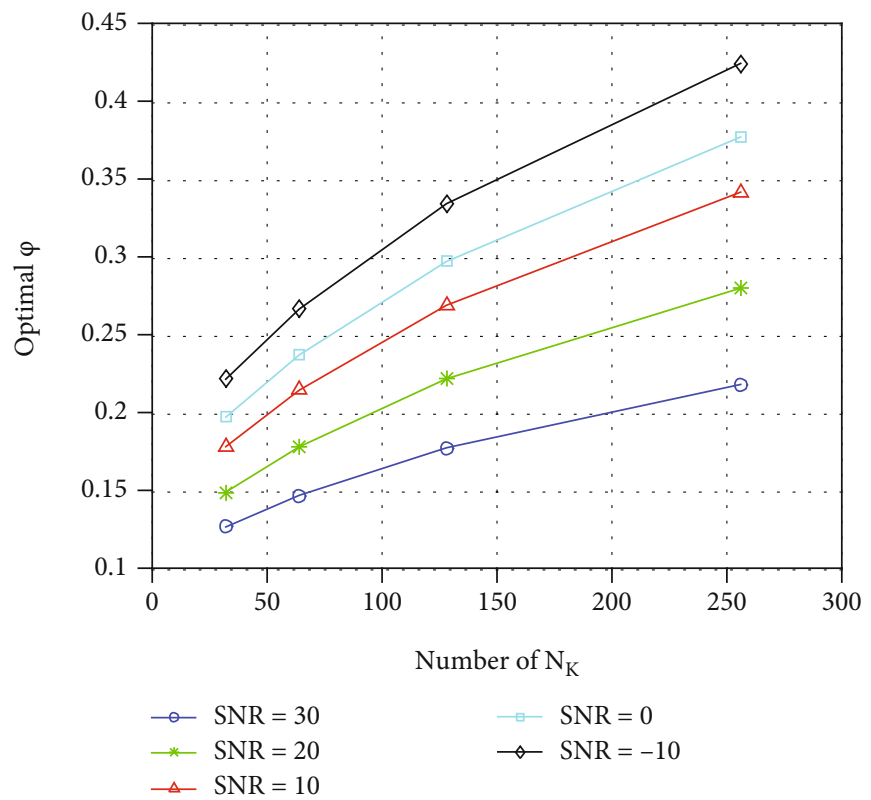

FIgURE 10: The optimal power distribution coefficient versus SNR with different $N_{L}$.

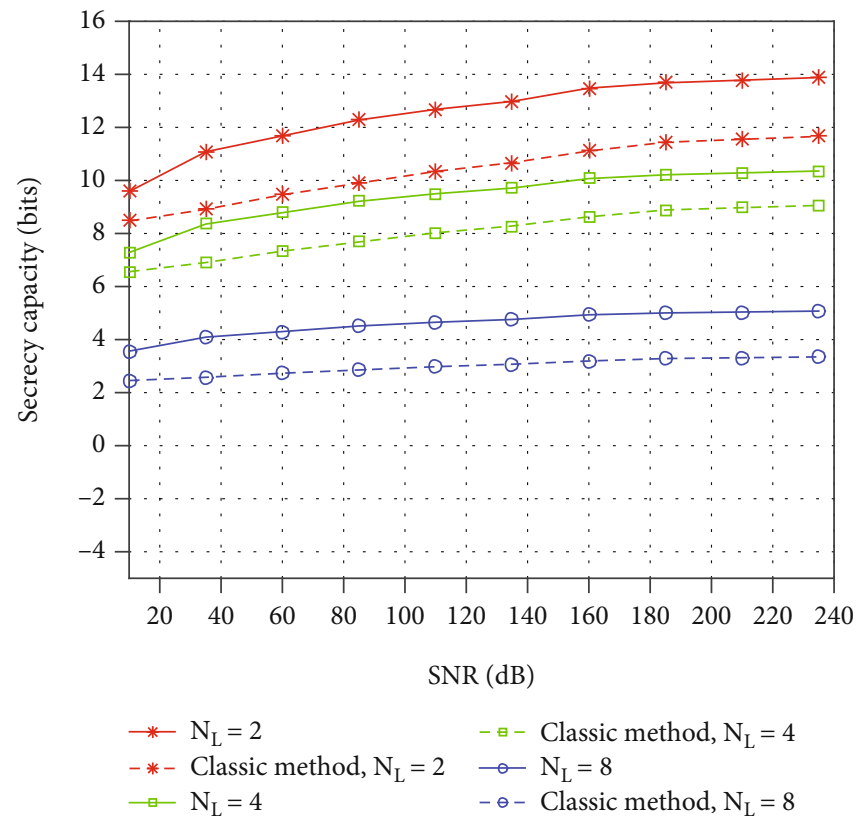

FIgURE 11: Comparison of SC between the algorithm in this paper and the algorithm in the classic papers [30].

Figure 8 shows the ELB of SC with different PA methods. We use SQP and BPA algorithms [26] to compare with the optimal PA coefficient presented in this paper. We can see from Figure 8 that under the condition that transmitting antennas' number is small, the ELB of SC using the optimal power distribution coefficient is smaller than that of SQP; when the number of antennas increases, the SC using the optimal power distribution coefficient increases significantly, which is consistent with the theory in this paper. Since the conclusions derived in this paper are derived using the strong law of large numbers, which is a statistical theory, the larger the number of samples, the more accurate the results of the theoretical derivation. Therefore, in the case of a small number of antennas, the algorithm in this paper is not as good as the traditional iterative optimization algorithm. But when the number of antennas is large enough, the algorithm in this paper is far better than the traditional iterative optimization algorithm. Since the optimal power distribution coefficient is based on statistics, the optimal power distribution coefficient can be used well for massive MIMO.

Figures 9 and 10 show optimal power distribution coefficient in (28) with different $N_{K}$ and $N_{L}$. Figures 9 and 10 show 
optimal $\phi$ reduces as $N_{L}$ grows; we should allocate more power to SCO-AN as $N_{L}$ increases. On the contrary, when $N_{K}$ increases, less power should be allocated to SCO-AN. Figures 9 and 10 also show that we should generate more power to the information-bearing signal with the SNR increase.

Figure 11 shows the comparison between the algorithm in this paper and the algorithm in the classic paper [30]. The solid line SC in Figure 11 is calculated by the optimal PA coefficient obtained by (28). The dotted SC is calculated by the best PA coefficient obtained in the classic paper [30]. Under the same conditions of $N_{k}$ and $N_{L}$, the algorithm in this paper has a better improvement on SC, while the algorithm in the classic paper [30] discards too many items, resulting in a significant error in the optimal PA coefficient, and the effect of improving the SC is reduced.

\section{Conclusion}

This paper researches ELB of SC under large-scale MIMO. Aiming at the problem that traditional optimization algorithms cannot find the maximum value of nonconvex PA function, this paper uses statistical methods to study the PA technology of SCO-AN in large-scale MIMO systems. First, this paper derives the expression of ELB of SC. Then, the best PA coefficient function based on this expression of ELB of SC is derived. According to the optimal PA coefficient function, the optimal PA coefficient under the conditions of different transmitting antennas and the number of eavesdropping antennas can be deduced. The application of the PA coefficient can significantly improve the SC without the optimization algorithm. This paper also considers the impact of imperfect CSI on PA; when $N_{K}$ increases or $N_{L}$ decreases, the power of SCO-AN should be reduced. At the same time, when the level of imperfect CSI increases, more power should be allocated to SCO-AN.

\section{Abbreviations \\ AN: $\quad$ Artificial noise \\ SCO-AN: Secrecy capacity optimization artificial noise \\ MIMO: Multiple-input multiple-output \\ CSI: Channel state information \\ SC: $\quad$ Secrecy capacity \\ PA: $\quad$ Power allocation \\ ELB: $\quad$ Ergodic lower bound \\ IRS: Intelligent reflecting surface.}

\section{Data Availability}

The datasets used and/or analyzed during the current study are available from the corresponding author on reasonable request.

\section{Conflicts of Interest}

The authors declare that they have no conflicts of interest.

\section{References}

[1] T. Hashem and R. Hasan, "Method and system for secure transmission of information," 2004, US Patent 6-792544.

[2] A. Wyner, "The wire-tap channel," Bell System Technical Journal, vol. 54, no. 8, pp. 1355-1387, 1975.

[3] I. Csiszar and J. Korner, "Broadcast channels with confidential messages," IEEE Transactions on Information Theory, vol. 24, pp. 339-348, 2003.

[4] J. Barros and M. Rodrigues, "Secrecy capacity of wireless channels," in 2006 IEEE international symposium on information theory, pp. 356-360, Seattle, 2006.

[5] F. Oggier and B. Hassibi, "The secrecy capacity of the MIMO wiretap channel," IEEE Transactions on Information Theory, vol. 57, no. 8, pp. 4961-4972, 2011.

[6] J. Zhu, S. Robert, and K. Vijay, "Linear precoding of data and artificial noise in secure massive MIMO systems," IEEE Transactions on Wireless Communications, vol. 15, pp. 2245-2261, 2015.

[7] M. Zeng, "Securing downlink massive MIMO-NOMA networks with artificial noise," IEEE Journal of Selected Topics in Signal Processing, vol. 15, pp. 685-699, 2019.

[8] M. Jo, H. Gao, Y. Su, S. Zhang, and Y. Hou, "Joint antenna selection and power allocation for secure co-time cofrequency full-duplex massive mimo systems," IEEE Transactions on Vehicular Technology, vol. 70, pp. 655-665, 2021.

[9] Y. Wu, R. Schober, D. W. K. Ng, C. Xiao, and G. Caire, "Secure massive MIMO transmission with an active eavesdropper," IEEE Transactions on Information Theory, vol. 62, no. 7, pp. 3880-3900, 2016.

[10] E. Ekrem and S. Ulukus, "The secrecy capacity region of the Gaussian MIMO multi-receiver wiretap channel," IEEE Transactions on Information Theory, vol. 57, no. 4, pp. 2083-2114, 2011.

[11] D. Ciuonzo, A. Aubry, and V. Carotenuto, "Vincenzo. C, Rician MIMO channel-and jamming-aware decision fusion," IEEE Transactions on Signal Processing, vol. 65, no. 15, pp. 3866-3880, 2017.

[12] J. Chakravarty, O. Johnson, and R. Piechocki, “A convex scheme for the secrecy capacity of a MIMO wiretap channel with a single antenna eavesdropper," in 2019 IEEE International Conference on Communications (ICC), pp. 1-5, Shanghai, 2019.

[13] Y. Jia, Z. Zhong, and F. Gao, "On the conditioning channel capacity of MIMO two-way relay networks against eavesdropper," in 10th International Conference on Communications and Networking in China, pp. 199-204, Shanghai, 2015.

[14] X. He and A. Yener, "MIMO wiretap channels with arbitrarily varying eavesdropper channel states," IEEE Transactions on Information Theory, vol. 60, pp. 6844-6869, 2017.

[15] R. Negi and S. Goel, "Secret communication using artificial noise," in IEEE Vehicular Technology Conference, p. 1906, Dallas, 2005.

[16] G. Yebo, W. Zhilu, Y. Zhendong, and Z. Xiaojun, “The secrecy capacity optimization artificial noise: a new type of artificial noise for secure communication in MIMO system," IEEE Access, vol. 7, pp. 58353-58360, 2019.

[17] X. Guan, Q. Wu, and Z. Rui, "Intelligent reflecting surface assisted secrecy communication: is artificial noise helpful or not?," IEEE Wireless Communications Letters, vol. 9, pp. 778-782, 2020. 
[18] X. Zhang, D. Guo, K. An, W. Ma, and K. Guo, "Secure transmission and power allocation in multiuser distributed massive MIMO systems," Wireless Networks, vol. 26, no. 2, pp. 941954, 2020.

[19] S. Hong, C. Pan, H. Ren, K. Wang, and A. Nallanathan, “Artificial-noise-aided secure MIMO wireless communications via intelligent reflecting surface," IEEE Transactions on Communications, vol. 68, no. 12, pp. 7851-7866, 2020.

[20] Y. Gu, B. Huang, and Z. Wu, "Power allocation for secrecy-capacity-optimization-artificial-noise secure MIMO precoding systems under perfect and imperfect channel state information," Apply Sciences, vol. 11, no. 10, p. 4558, 2021.

[21] F. Khan, Z. Malik, A. Nasir, and M. Masood, "Relay selection \& power allocation for maximizing sum-throughput of a buffered relay network," IEEE Communications Letters, vol. 99, pp. 1318-1322, 2020.

[22] J. Zhu, "On optimal power allocation for downlink nonorthogonal multiple access systems," IEEE Journal on Selected Areas in Communications, vol. 35, pp. 2744-2757, 2017.

[23] H. Zhang, "Downlink energy efficiency of power allocation and wireless backhaul bandwidth allocation in heterogeneous small cell networks," IEEE Transactions on Communications, vol. 66, pp. 1705-1716, 2017.

[24] M. Chen and A. Yener, "Power allocation for F/TDMA multiuser two-way relay networks," IEEE Transactions on Wireless Communications, vol. 9, no. 2, pp. 546-551, 2010.

[25] Y. Jiang, Y. Zou, H. Guo, J. Zhu, and J. Gu, "Power allocation for intelligent interference exploitation aided physical-layer security in ofdm-based heterogeneous cellular networks," IEEE Transactions on Vehicular Technology, vol. 69, no. 3, pp. 3021-3033, 2020.

[26] C. Büskens and H. Maurer, "SQP-methods for solving optimal control problems with control and state constraints: adjoint variables, sensitivity analysis and real-time control," Journal of Computational and Applied Mathematics, vol. 120, no. 1-2, pp. 85-108, 2000.

[27] D. Tse, "Optimal power allocation over parallel Gaussian broadcast channels," in Proceedings of IEEE International Symposium on Information Theory, p. 27, Ulm, Germany, 1997.

[28] Y. Zhao, A. Raviraj, and J. Teng, "Improving amplify-andforward relay networks : optimal power allocation versus selection," in IEEE International Symposium on Information Theory, pp. 1234-1238, Seattle, 2006.

[29] Z. Ding, S. M. Perlaza, I. Esnaola, and H. V. Poor, "Power allocation strategies in energy harvesting wireless cooperative networks," IEEE Transactions on Wireless Communications, vol. 13, no. 2, pp. 846-860, 2014.

[30] X. Zhou and R. Matthew, "Secure transmission with artificial noise over fading channels: achievable rate and optimal power allocation," IEEE Transactions on Vehicular Technology, vol. 59, no. 8, pp. 3831-3842, 2010.

[31] H. Gao, P. Smith, and V. Clark, "Theoretical reliability of MMSE linear diversity combining in Rayleigh-fading additive interference channels," IEEE Transactions on Communications, vol. 46, no. 5, pp. 666-672, 1998.

[32] M. Abramowitz, I. Stegun, and I. Romain, "Handbook of Mathematical Functions, with Formulas, Graphs, and Mathematical Tables," Physics Today, vol. 19, no. 1, pp. 120-121, 1966. 